\title{
Intestinal Microbiota and Its Link with Mind and Heart
}

\section{ISSN: 2637-7632}

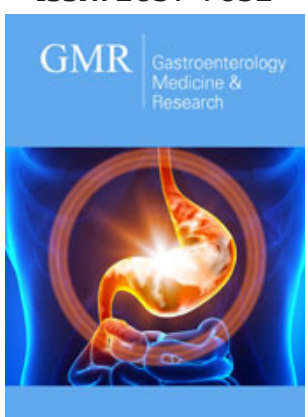

*Corresponding author: Álvaro Zamudio Tiburcio, Department of Gastroenterology, Intestinal Microbiota Transplantation Hospital Trinidad, Mexico

Submission: 漹 September 27, 2019

Published: 㭗October 11, 2019

Volume 3 - Issue 5

How to cite this article: Álvaro Zamudio Tiburcio, Héctor Bermúdez Ruiz, Pedro Antonio Reyes López. Intestinal Microbiota and Its Link with Mind and Heart. Gastro Med Res. 3(5). GMR.000571. 2019.

DOI: 10.31031/GMR.2019.03.000571

Copyright@ Álvaro Zamudio Tiburcio, This article is distributed under the terms of the Creative Commons Attribution 4.0 International License, which permits unrestricted use and redistribution provided that the original author and source are credited.

\author{
Álvaro Zamudio Tiburcio ${ }^{1 *}$, Héctor Bermúdez Ruiz ${ }^{2}$ and Pedro Antonio Reyes \\ López ${ }^{3}$ \\ ${ }^{1}$ Department of Gastroenterology, Mexico \\ ${ }^{2}$ Department of Endoscopy, Mexico \\ ${ }^{3}$ Department of Cardiology, Mexico
}

\begin{abstract}
Summary
A mini review about how the Intestinal Microbiota (IM) is linked to the mind and heart is carried out. It is pointed out how once IM inflammation (Dysbiosis) generated, a series of mechanisms, immunological, neuronal and endocrine, that affect host phenotypes are triggered. The interpretation of what the gutmicrobiota-brain axis is deepened. (GBA), and it is exemplified how, through bidirectional communications with the Intestinal Microbiota, a series of changes, which impact human health, are translated. Likewise, 10 conclusions are proposed on diverse topics, because the IM not only relates to the mind-heart binomial, but it has to do with many organs and consequently with many sufferings.
\end{abstract}

Keywords: Microbiome; Intestine-microbiota-brain axis (GMB); Intestinal microbiota transplantation (IMT); Mind-heart

\section{Commentary}

Today, various functions that the Microbiota exerts have been better understood; Above all, the Intestinal Microbiota, since this is the most significant of the Microbiota, because it represents the highest percentage of it. It's in the intestine, especially in the colon, where the IM lives, and where a series of conversations take place, where microorganisms and environmental antigens interact with the host and, translate, the magnificent functions of this super-organ. Among these functions are protection, against pathogens, metabolic reactions, stimulation of immunological processes, homeostasis, the collection of ingested energy, the generation of vitamins, among many others [1]. IM not only links with the mind, it also does it with the heart, through signals similar to those produced by hormones, by affecting the host's metabolic phenotypes. There are also direct impacts of metabolites on the development of thrombosis. Finally, some Acids Short chain fats, produced in the microbial fermentation of complex carbohydrates, may increase the risk of vascular brain disease [2-4]. It has been reported that antibiotic-generated dysbiosis stimulates the release of cytokines, which aggravate the cardiac lesion, in rats, with major ischemia and myocardial re-perfusion [5]. To understand all of the above, we must go where all these transmissions are generated. That place is called the gut-microbiota-brain axis. There are interactive and parallel channels that communicate the intestinal microbiota with the brain. These channels are both endocrine, neuronal and immunological and, the brain, through the Autonomous Nervous System, interacts in the intestinal secretion, its transit; its motility, as well as its permeability, translating diverse manifestations of the genome of the microorganisms, existing there. All this communication is a circuit, where there will be clinical manifestations, depending on the place of involvement [6]. In other way, gastrointestinal and psychiatric conditions are associated with GBA e, influenced by environmental and genetic factors. Due to the above, the challenge of the next generation is to detect, why and in what part of this transmission, there is an affectation; Once this problem is elucidated, the cure of both mental and gastrointestinal diseases will be closer [7]. Now, how does the effects of certain medications alter the intestinal microbiota, and consequently, its impact on health?

One of the first examples that was pointed out, is the synthesis of bile acids in secondary bile acids, in the liver gland, through the competition of the intestinal microbiota. The same thing happens in numerous medications such as L-dopa, digoxin, carnitine, acetaminophen, etc. Its concluded that medications and diet impact the health of the Intestinal Microbiota, 
which translates into dysbiosis. And finally, in psychological, gastrointestinal, skin conditions, etc [8]. Not only does Microbiota Disease affect the mind and heart, but it does so in other organs, such as the liver. In it, hepatocellular cancer, steato-hepatitis, non-alcoholic fatty liver and other conditions may appear, so it is recommended to rationalize the administration of antibiotics and some other toxic components that affect the liver [9]. A sufferings of the mind as it is, Autism Spectrum Disorder (ASD), has been determined by Microbiota Disease. The inflammation of this superorgan generates this complex human behavior, as has been observed, in mice transplanted with intestinal microbiota, from patients with ASD [10]. Evidence has continued to appear that dysbiosis is closely related to the mind-heart binomial and bidirectional signals have been present in this interesting communication.

Not only has it been written about conventional disorders of the effect of the microbiota, we also read articles of transplanted patients, as a final methodology, in terminal disease. Due to the above and the complications inherent to these methods, IM has been studied and, significant changes have been observed in transplanted patients, so these changes can be used as prognosis. Concomitantly, they have turned their eyes to the Intestinal Microbiota Transplantation (IMT) and, to the use of prebiotics and probiotics in this type of patient [11]. And not only has Intestinal Microbiota Transplantation been explored in donors without special diets, but it has been done in Beganos, with flattering results [12]. Its evident that Intestinal Microbiota Transplantation (IMT) improves numerous neurological problems, through the correction of dysbiosis. It has been considered that this occurs, provided that the functions of immune cells and brain drainage are not compromised [13].

\section{Conclusion}

1. The intestinal microbiota, boosts host metabolism; confers resistance to infections, inflammatory processes, the development of neoplasms and autoimmunity. It promotes endocrine functions and collaborates with neurological function, through the so-called "gut-microbiota-brain axis" [14].

2. IM is linked to the mind and heart, through signals similar to those produced by hormones, by affecting the host's metabolic phenotypes [15].

3. Intestinal Microbiota Dysbiosis generated by antibiotics stimulates the release of cytokines, which aggravate the cardiac lesion [16].

4. On the intestine-microbiota-brain axis, there are interactive and parallel channels, which communicate the Intestinal Microbiota with the brain [17].

5. The cure of both mental and gastrointestinal diseases will be closer, when detected, why and where in the transmission of GBA, there is involvement [18].

6. The Intestinal Microbiota Disease affects many other organs, such as the liver, so it's recommended to rationalize the administration of antibiotics and some other toxic components that affect the liver gland [19].

7. Autism Spectrum Disorder has been determined, which is generated by Intestinal Microbiota Disease [20].

8. They have turned their eyes, towards the Intestinal Microbiota Transplant and, to the use of prebiotics and probiotics, in transplanted patients [21].

9. Flattering results have been observed in Beganos, which has been used as donors of Intestinal Microbiota [22].

Intestinal Microbiota Transplantation improves numerous neurological problems, by correcting dysbiosis [23]..

\section{References}

1. Montalvo M, Onofrio FD, Gallo A, Cazzato A, Gasbarrini G (2009) Intestinal microbiota and its functions. Digestive and Liver Disease Supplements 3(2): 30-34.

2. McMillan A, Hazen SL (2019) Gut microbiota involvement in ventricular remodeling post-myocardial infarction. Circulation 139(5): 660-662.

3. Zhu W, Gregory JC, Org E, Buffa JA, Gupta N, et al. (2016) Gut microbial metabolite TMAO enhances platelet hyperreactivity and thrombosis risk. Cell 165(1): 111-124.

4. Klaassen CD, Cui JY (2015) Mechanisms of how the intestinal microbiota alters the effects of drugs and bile acids. Drug Metab Dispose 43(10): 1505-1521.

5. Lam V, Su J, Koprowski S, Hsu A, Tweddell JS, et al. (2012) Intestinal microbiota determine severity of myocardial infarction in rats. FASEB J 26(4): 1727-35.

6. Martin CR, Osadchiy V, Kalani A, Mayer EA (2018) The Brain-GutMicrobiome axis. Cell Mol Gastroenterol Hepatol 6(2): 133-148.

7. Zydecka SK, Marlicz W, Misera A, Koulaouzidis A, Loniewski I (2018) Microbiome-the missing link in the gut-brain axis: Focus on its role in gastrointestinal and mental health. J Clin Med 7(12): 521.

8. Hadrich D (2018) Microbiome research is becoming the key to better understanding health and nutrition. Front Genet 9: 212.

9. Hagymási K, Bacsárdi A, Egresi A, Berta E, Tulassay Z, et al. (2018) The role of gut microbiota in chronic liver diseases, and treatment possibilities. Orv Hetil 159(36): 1465-1474.

10. Sharon G, Cruz NJ, Kang DW, Gandal MJ, Wang B, et al. (2019) Human gut microbiota from autism spectrum disorder promote behavioral symptoms in mice. Cell 177(6): 1600-1618.

11. Spinner JA, Denfield SW, Luna R, Balderas M, Dreyer WJ, et al. (2018) Alteration of the intestinal microbiome in pediatric hearth transplant patients is associated with diarrhea. J Pediatr Hearth Transpl 37(4): S64.

12. Smits LP, Kootte RS, Levin E, Prodan A, Fuentes S, et al. (2018) Effect of vegan fecal microbiota transplantation on carnitine- and cholinederived Trimethylamine-N-Oxide production and vascular inflammation in patients with Metabolic Syndrome. JAHA 7(7): e008342.

13. Ruggiero M (2016) Fecal microbiota transplantation and the brain microbiota in neurological diseases. Clin Endosc 49(6): 579.

14. Kataoka K (2016) The intestinal microbiota and its role in human health and disease. J Med Invest 63(1-2): 27-37.

15. Qianquan M, Xing C, Long W, Wang HY, Qing L, et al. (2019) Impact of microbiota on central nervous system and neurological diseases: the gut-brain axis. J Neuroinflammation 16(1): 53.

16. Yoon MY, Yoon SS (2018) Disruption of the gut ecosystem by antibiotics. Yonsei Med J 59(1): 4-12. 
17. Carabotti M, Scirocco A, Maselli MA, Severi C (2015) The gut-brain axis: interactions between enteric microbiota, central and enteric nervous systems. Ann Gastroenterol 28(2): 203-209.

18. Galland L (2014) The gut microbiome and the brain. J Med Food 17(12): 1261-1272.

19. Tilg H, Cani PD, Mayer EA (2016) Gut microbiome and liver diseases. Gut 65(12): 2035-2044.

20. Liu F, Li J, Wu F, Zheng H, Peng AP, et al. (2019) Altered composition and function of intestinal microbiota in autism spectrum disorders; a systematic review. Transl Psychiatry 9: 13-43.
21. Jorgenson MR, Descourouez JL, Siodlak M, Tjugum S, Rice JP, et al. (2018) Efficacy and safety of probiotics and symbiotics in liver transplantation. Pharmacotherapy.

22. Porras D, Nistal E, Martinez-Flórez S, Olcoz JL, Jover R, et al (2019) Functional interactions between gut microbiota transplantation, quercetin, and high-fat diet determine non-alcoholic fatty liver disease development in germ-free mice. Mol Nutr Food Res 63(8): e1800930.

23. Griffiths JA, Mazmanian SK (2018) Emerging evidence linking the gut microbiome to neurologic disorders. Genome Med 10(1): 98. 